\title{
Ultrasound appearance of preclinical Oesophagostomum bifurcum induced colonic pathology
}

Background-Oesophagostomum bifurcum is a parasitic helminth endemic in southern West Africa, infecting an estimated 250000 people. ${ }^{12}$ It develops within the colonic wall resulting in symptomatic granulomatous lesions detectable by ultrasound, ${ }^{3}$ and accounts for $1 \%$ of major surgeries within the endemic area of northern Ghana, presenting with peritonitis or bowel obstruction. ${ }^{4}$ These echo free lesions within the abdominal or colonic wall of $50 \mathrm{~mm}$ in diameter, on average, have a well defined ovoid shape and are surrounded by a poorly reflective wall. They protrude into the colonic lumen and show posterior wall enhancement.

However, asymptomatic abdominal lesions of smaller but similar appearance are commonly observed on ultrasound in healthy individuals from villages within this area. $^{5}$ The lesions are suspected to have $O$ bifurcum aetiology.

Case reports-We observed two patients from northern Ghana presenting with right lower quadrant (RLQ) abdominal pain indicative of appendicitis. As apparently incidental asymptomatic findings in both cases, suspected $O$ bifurcum induced echo free lesions were detected on the transverse colon. We present the case history of one of these patients.

An eight year old female from the Bimoba ethnic group presented with a two week history of intermittent RLQ abdominal pain and decreased appetite. Three days before hospital admission the pain became severe and constant, and was associated with fever.

On ultrasound examination, an indistinct poorly reflective lesion consistent with the appearance of an abscess was observed in the RLQ. Routine colonic investigation revealed an echo free lesion $17 \mathrm{~mm}$ in diameter which protruded into the lumen of the proximal transverse colon adjacent to the gall bladder (fig 1). The lesion was identical to the suspected $O$ bifurcum induced lesions commonly observed in healthy individuals. The transverse colon and

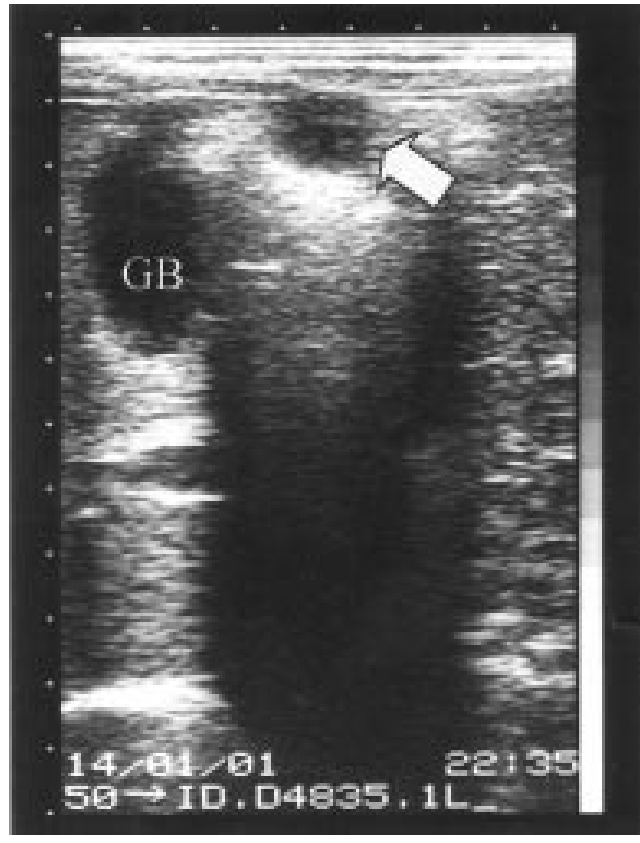

Figure 1 Transverse view of the hepatic flexure: echo free lesion within the highly reflective colon adjacent to the gall bladder $(G B)$. lesion were freely moving with respiration, indicating that neither were adhered to the abdominal wall.

During emergency exploratory

laparotomy, a thick walled $(5 \mathrm{~mm})$ structure $60 \mathrm{~mm}$ in diameter within the wall of the proximal ascending colon was found to be adhered to the lateral abdominal wall. A perforation occurred during dissection of the lesion from the parietal peritoneum and thick yellow pus was visualised. The appendix was normal, and no cause for the abscess could be found.

In response to the ultrasound finding in the transverse colon, the hepatic flexure was also inspected. Within the subserosa, there was a hard nodule of $20 \mathrm{~mm}$ in diameter (fig 2). It was not surrounded by adhesions, nor was it adherent to the abdominal wall or to other bowel sections. The colon proximal to the nodule was of normal diameter and appearance. Approximately $5 \mathrm{ml}$ of thick yellow pus were released by a small incision through the $2 \mathrm{~mm}$ thick wall. The nodule was further explored, and no communication between it and the colonic lumen was found. A white worm $1 \mathrm{~cm}$ long was removed alive from the pus, and it was later identified as being a juvenile non-gravid female O bifurcum.

Albendazole $200 \mathrm{mg}$ daily for five days was prescribed. The immediate postoperative recovery was uneventful, but after discharge the patient was lost to follow up.

The second case, a 15 year old male of the Bimoba ethnic group, mirrored the one presented.

Discussion - The lesions commonly noted in healthy individuals from villages within northern Ghana $^{5}$ are identical to those seen in these two patients, and we therefore suggest that the preclinical pathology of $O$ bifurcum has this characteristic ultrasound appearance. The ability to make a preclinical diagnosis of oesophagostomiasis will permit attempts at early pharmacological intervention. Oesophagostomiasis can give rise to severe surgical emergencies ${ }^{4}$ and it therefore merits a place in the awareness of both clinicians in the tropics and those practising in areas of the world to whom tropical travellers consult.

Edinburgh University Medical School,

P A STOREY

Teviot Place, Edinburgh EH8 $9 A G, U K$

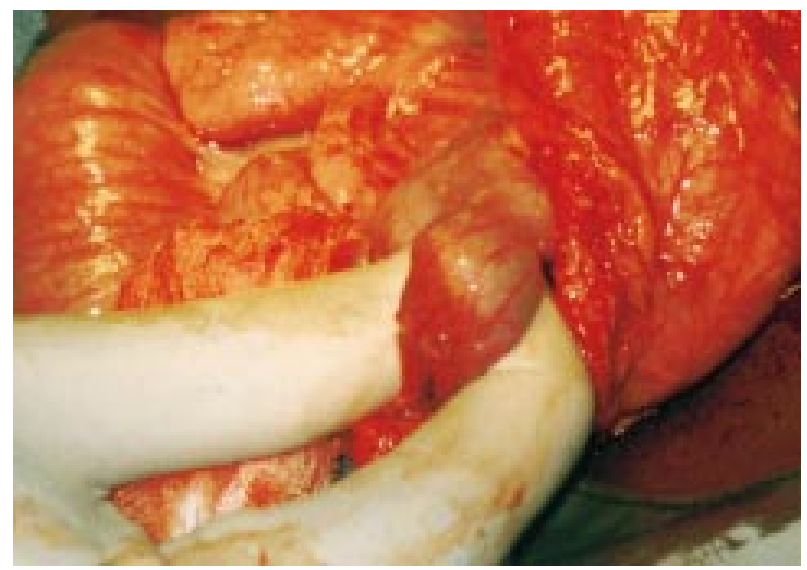

Figure 2 Hard nodule of $2 \mathrm{~cm}$ in diameter in the subserosa of the hepatic flexure. 
Nalerigu Baptist Medical Centre, Box 50 Gambaga, Northern Region, Ghana

Radiology Department, Leiden University Medical Centre, PO Box 9600, 2300RC Leiden, the Netherlands

Ministry of Health, PO Box 99 Tamale, Northern Region, Ghana

P A STOREY A M POLDERMAN

Parasitology Department, Leiden University Medical Centre, PO Box 9600, 2300RC Leiden, the Netherlands

P MAGNUSSEN

Danish Bilharziasis Laboratory,

faegersborg Alle 1D, Charlottenlund DK 2920, Denmark

Correspondence to: Dr A M Polderman. A.M.Polderman@LUMC.nl
G FAILE The Leverhulme Trust, the Sir Halley Stewart Trust, the Danish Bilharziasis Laboratory, and Leiden University Parasitology Department provided financial contributions enabling this study and the ongoing project. The help of Banle Seidu who translated the histories was invaluable. The support of the staff from the Baptist Medical Centre, Nalerigu, to whom the patients presented, is gratefully acknowledged.

1 Krepel HP, Baeta S, Polderman AM. Human oesophagostomum infection in northern Togo and Ghana: epidemiological aspects. Ann Trop Med Parasitol 1992;86:289-300.

2 Polderman AM, Anemana S, Asigri V. Human oesophagostomiasis: a regional public health problem in Africa. Parasitol Today 1999;15:129-30. 3 Storey PA, Anemana S, van Oostayen JA, et al. Ultrasound diagnosis of oesophagostomiasis. Br f Radiol 2000;73:328-32.

4 Storey PA, Faile G, Hewitt E, et al. Clinical epidemiology and classification of human oesophagostomiasis. Trans R Soc Trop Med Hyg 2000;94:17782 .

5 Storey PA, Spannbrucker N, Yelifari L, et al. Ultrasound detection and assessment of pre-clinical Oesophagostomum bifurcum induced colonic pathology. Clin Infect Dis (in press). 